communication provides further evidence of the essential part played by calcium in isoprenaline-induced relaxation of the depolarized rat uterus.

When an isolated rat uterus which has been depolarized by immersion in sodium-free potassium chloride- or potassium sulphate-Ringer is treated with isoprenaline it relaxes, but if a calcium chelating agent such as EDTA (ethylenediamine tetraacetic acid) or the more specific EGTA (ethyleneglycolbis - aminoethylether - tetraacetic acid) has previously been added to the solution no relaxa. tion occurs with isoprenaline ${ }^{6}$. Chelating agents also abolish papaverine-induced relaxation. These effects of chelation seem to suggest that the removal of calcium from a strategic site has rendered the relaxant drugs in. effective, but because chelating agents themselves relax the depolarized uterus it could be objected that the failure of isoprenaline to relax further is simply due to the contractile element being already fully extended. This objection is disproved by the following experiment (Fig. 1).

The two horns of an isolated rat uterus were suspended in calcium-free potassium chloride-Ringer ( $145 \mathrm{mmolar}$ potassium chloride, 12 mmolar potassium bicarbonate, 6 mmolar glucose) and treated with $10^{-3}$ molar EGTA until they ceased to respond to isoprenaline. At this point calcium was added to the bathing solution of one horn and barium to the other, whereon both horns shortened maximally. After readjustment to the original tension isoprenaline was applied which then produced a relaxation in the horn treated with calcium but none in the horn treated with barium. The experiment was then repeated in a crossover arrangement with identical results.

The finding that isoprenaline relaxes the muscle contracted by the calcium but not that contracted by the barium suggests that this relaxation involves a mechanism which is specifically adapted to calcium. (In this connexion, it is of interest that I have found barium also to be a rather inefficient substitute for calcium in supporting the contractile effect of acetylcholine in the depolarized muscle.) It is a possibility that calcium is removed from the contractile element during isoprenaline relaxation and the question arises whether it is then taken up by an intracellular storage site or extruded into the extracellular space. An intracellular process seems functionally more probable in spite of the lack of a prominent endoplasmic reticulum in smooth muscle ${ }^{7}$; thus it has been found that isoprenaline relaxes the depolarized smooth muscle irres. pective of the magnitude of the external calcium concentration $^{6}$. An attractive hypothesis is that regional intracellular calcium shifts of opposite sign underlie contraction by acetylcholine and relaxation by isoprenaline of the depolarized uterus.

H. O. SCHILD

Department of Pharmacology,

University College, London.

Received April 4, 1967.

Robertson, P. A., Nature, 186, 316 (1960).

2 Yukishada, N., and Ebashi, F., Jap. J. Pharmacol.,11, 46 (1961).

${ }^{3}$ Durbin, R. P., and Jenkinson, D. H., J. Physiol., 157, 90 (1961).

- Edman, K. A. P., and Schild, H. O., J. Physiol., 161, 424 (1962).

${ }^{5}$ (hujyo, N., and Holland, W. C., Amer. J. Physiol., 205, 94 (1963).

6 Schild, H. O., Pharmacol. Rev., 18, 495 (1966).

${ }^{7}$ Hasselbach, W., Prog. Biophysics, 14, 167 (1964).

\section{Ionic Requirements of Synaptic Transmitter Release}

THe release of a transmitter substance by the nerve impulse depends on the presence of calcium, rather than sodium, on the outside of the axon membrane. There is increasing evidence that inward movement of calcium is one of the first steps in the "electro-secretory" process. This view has been strengthened by recent experiments with tetrodotoxin ${ }^{1-3}$, which eliminates the regenerative
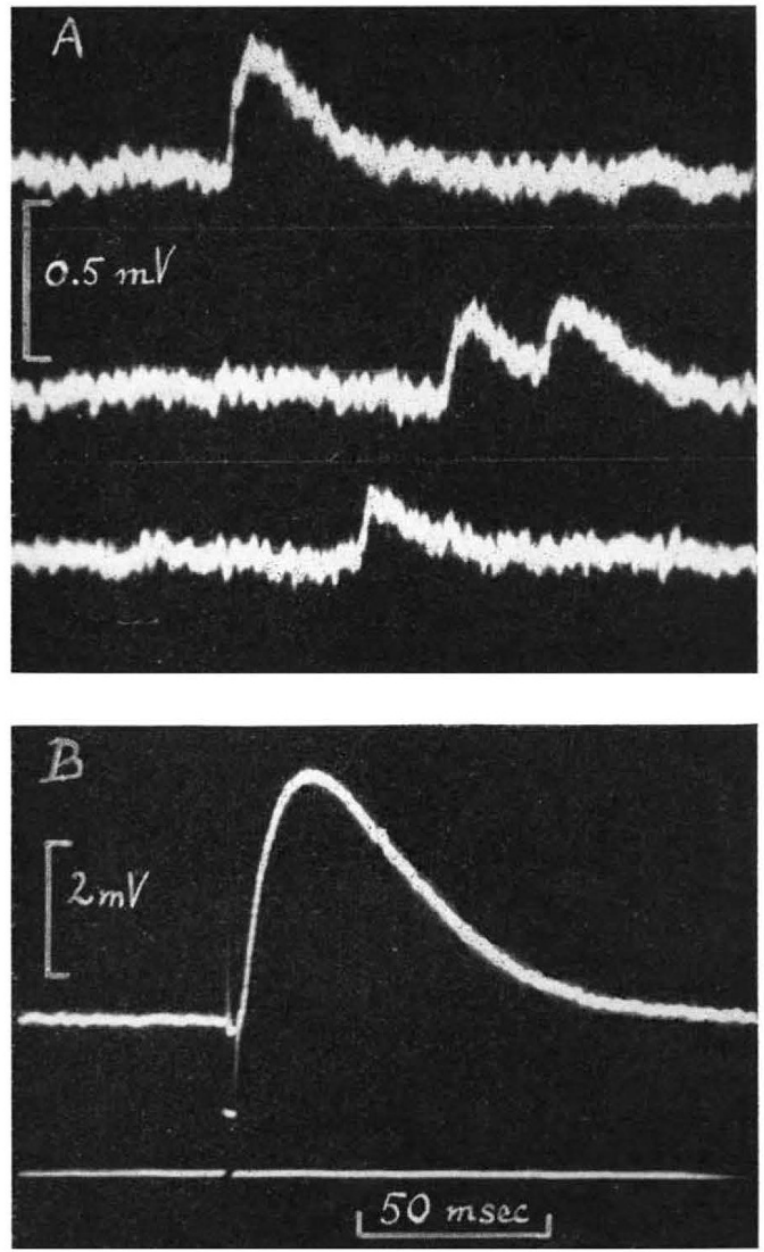

Fig. 1. Intracellular records of spontaneous miniature end-plate potentials $(A)$, and evoked end-plate potential $(B)$, from a frog sartorius kept in "calcium Ringer". Temperature $6.5^{\circ} \mathrm{C}$. The lower trace in $B$ shows the depolarizing current pulse (about $2 \mathrm{msec}$ ) applied to the nerve ending.

influx of sodium ions but does not interfere with the release of the transmitter by locally imposed depolarization provided calcium ions are present in the external solution.

Further evidence was obtained in an experiment in which a frog sartorius muscle had been equilibrated in a "sodium-free Ringer" containing 83 mmolar calcium chloride, 2 mmolar potassium chloride, and $2 \times 10^{-6}$ $\mathrm{g} / \mathrm{ml}$. of neostigmine methylsulphate. When intracellular records were obtained from junctional regions of muscle fibres, spontaneous miniature end-plate potentialsthough much reduced in size-could still be recorded (Fig. $1 A$ ). When the nerve ending was depolarized locally, by passing negative going pulses through a closely applied micro-pipette (filled with 1 molar calcium choride), endplate potentials were elicited similar to those previously observed in the tetrodotoxin experiments ${ }^{1}$ (Fig. $1 B$ ).

Thus release of acetylcholine in response to membrane depolarization occurs in the absence of external sodium, when the sodium ions in the bath have been totally replaced by calcium.

\section{B. KaTz}

R. Mixedi

Department of Biophysics,

University College London.

Received June 29, 1967.

1 Katz, B., and Miledi, R., Nature, 20\%, 1097 (1965).

2 Katz, B., and Miledi, R., Proc. Roy. Soc., B, 167, 23 (1967).

${ }^{3}$ Katz, B., and Miledi, R., Nature, 212, 1242 (1966). 